\title{
Evaluation of contrast-enhanced ultrasonography for hepatocellular carcinoma prior to and following stereotactic body radiation therapy using the CyberKnife ${ }^{\circledR}$ system: A preliminary report
}

\author{
KAZUE SHIOZAWA $^{1,2}$, MANABU WATANABE ${ }^{2}$, TAKASHI IKEHARA ${ }^{2}$, KOJIRO KOBAYASHI $^{1}$, \\ YUTA OCHI $^{1}$, YUTA SUZUKI ${ }^{1}$, KAZUHIRO FUCHINOUE $^{1}$, MASATAKA YONEDA ${ }^{1}$, \\ TAKESHI KENMOCHI ${ }^{1}$, YUSUKE OKUBO ${ }^{1}$, TAKAYUKI MORI ${ }^{1}$, HIROYUKI MAKINO ${ }^{1}$, \\ NOBUHIRO TSUKAMOTO ${ }^{3}$, YOSHINORI IGARASHI ${ }^{2}$ and YASUKIYO SUMINO ${ }^{2}$ \\ ${ }^{1}$ Division of Gastroenterology and Hepatology, Department of Internal Medicine, Saiseikai Yokohamashi Tobu Hospital, \\ Yokohama, Kanagawa 230-0012; ${ }^{2}$ Division of Gastroenterology and Hepatology, Department of Internal Medicine, \\ Toho University Medical Center, Omori Hospital, Tokyo 143-8541; ${ }^{3}$ Department of Radiology, \\ Saiseikai Yokohamashi Tobu Hospital, Yokohama, Kanagawa 230-0012, Japan
}

Received November 13, 2014; Accepted September 21, 2015

DOI: $10.3892 / 01.2015 .3874$

\begin{abstract}
The CyberKnife ${ }^{\circledR}$ is expected to be a novel local treatment for hepatocellular carcinoma (HCC), however, a long-term follow-up using dynamic computed tomography and magnetic resonance imaging is required to determine the effect of treatment in a number of the affected patients. Therefore, there is a requirement to evaluate procedures for early determination of the effect of CyberKnife treatment. The present study aimed to evaluate the changes in the hemodynamics of the tumors and the hepatic parenchyma surrounding the tumor prior to and following CyberKnife treatment for HCC. A total of $4 \mathrm{HCC}$ patients were enrolled in this study. These patients underwent CyberKnife treatment and were evaluated by image analysis prior to and following treatment using contrast-enhanced ultrasonography (CEUS) with Sonazoid. CEUS was performed prior to treatment, at 2 and 4 weeks post-treatment, and every 4 weeks thereafter for as long as possible. The dynamics of the enhancement of the tumor and the hepatic parenchyma surrounding the tumor in the vascular phase, and the presence or absence of a hypoechoic area in the hepatic parenchyma surrounding the tumor in the post-vascular phase were assessed. Results showed that: i) In
\end{abstract}

Correspondence to: Dr Manabu Watanabe, Division of Gastroenterology and Hepatology, Department of Internal Medicine, Toho University Medical Center, Omori Hospital, 6-11-1 Omorinishi, Ōta, Tokyo 143-8541, Japan

E-mail:manabu62@med.toho-u.ac.jp

Key words: hepatocellular carcinoma, contrast-enhanced ultrasonography, stereotactic body radiotherapy, CyberKnife ${ }^{\circledR}$, Sonazoid the patient with earlier changes, hemodynamic changes were evident in the tumor at 4 weeks and in the hepatic parenchyma surrounding the tumor at 2 weeks post-treatment, respectively; ii) the tumor showed hypoenhancement in all patients; and iii) with regard to findings in the hepatic parenchyma surrounding the tumor, strong hyperenhancement appeared in the vascular phase initially, followed by a hypoechoic area in the post-vascular phase. Evaluation of the hemodynamics of tumors and hepatic parenchyma surrounding the tumor using CEUS with Sonazoid may be therapeutically applicable, as it is less invasive than dynamic computed tomography (CT) and provides an early evaluation of the effectiveness of CyberKnife treatment.

\section{Introduction}

CyberKnife ${ }^{\circledR}$ (Accuray Inc., Sunnyvale, CA, USA) stereotactic body radiotherapy (SBRT) (1-4), an image-guided robotic radiosurgery system, is a radiation delivery platform that is capable of detecting and correcting for intrafraction tumor motion, as well as being able to adapt to the patient's breathing pattern and moving the linear accelerator in concert (1). CyberKnife was developed in the United States in 1992 (2), applied clinically in 1994 and introduced in Japan in 1997. CyberKnife can be used to perform multi-directional irradiation and disperse the dose among the normal tissues due to a high degree of freedom for the direction of the irradiation. Therefore, irradiation by CyberKnife treatment is more intensive than SBRT by conventional linac (5).

The therapeutic indications of CyberKnife previously included brain tumors, and head and neck cancer (3). However, therapeutic application against cancer in the trunk, including hepatocellular carcinoma (HCC), was begun following approval in June 2008 (4). Cancer in the trunk moves with respiration. However, CyberKnife detects minute 
body movements and fine-tunes the irradiation angle using a seeker (6). The technique is therefore expected to become a novel local treatment for HCC due to its minimal invasiveness and the reduced impact on patients (7-9).

CyberKnife treatment of HCC was introduced into Saiseikai Yokohamashi Tobu Hospital (Yokohama, Japan) in December 2011, and dynamic CT has mainly been used to determine its therapeutic effect. However, this technique could take $\geq 6$ months to observe a marked effect in a number of the affected patients. Therefore, it is expected that the establishment of a method that will allow the early determination of the effectiveness of treatment will improve the response.

We previously performed hemodynamic diagnoses of HCC and liver metastases, and evaluated the early responses to sorafenib for HCC using contrast-enhanced ultrasonography (CEUS) with Sonazoid, and confirmed their usefulness $(10,11)$. In the present study, CEUS was performed prior to and following CyberKnife treatment, and the changes in the images were evaluated to investigate whether CEUS can be applied therapeutically for the early determination of the effect of CyberKnife treatment.

\section{Materials and methods}

Indications of CyberKnife. In Saiseikai Yokohamashi Tobu Hospital, CyberKnife is applied to treat HCC patients with a performance status of $\leq 2$, a Child-Pugh score of A to 8-B, a total bilirubin level of $\leq 3 \mathrm{mg} / \mathrm{dl}$, an indocyanine green retention rate at $15 \mathrm{~min}$ of $\leq 50 \%$, no ascites, $\leq 3$ tumors with a tumor diameter of $\leq 3 \mathrm{~cm}$, a single tumor with a tumor diameter of $\leq 5 \mathrm{~cm}$, tumors $\geq 1 \mathrm{~cm}$ away from the intestine, and tumors with no connection to the gallbladder.

CyberKnife methodology. Since CyberKnife treatment of cancer in the trunk, including HCC, cannot be performed using the skeleton as the focal point, i.e., the skull in head and neck cancer, a gold fiducial marker (Toyo Medic Co., Tokyo, Japan) was installed prior to treatment as an ultrasound-guided target for percutaneous transhepatic radiation. The gold fiducial marker is a coiled device $(0.75 \mathrm{~mm}$ in diameter by $5 \mathrm{~mm}$ in length) that is implanted around the lesions. If a lesion was detected in the right or left lobe of the liver, the marker could then be implanted in the right or left lobe. The irradiation treatment plan was formed by CT following implantation of the target. The initial total irradiation dose was $60 \mathrm{~Gy}$ and the dose was increased or decreased based upon the tumor size, location and residual liver function, as required. Irradiation was divided into 3-5 fractions. The irradiation range of the hepatic parenchyma surrounding the tumors was $\geq 17$ Gy and the irradiated site was $\leq 20 \%$ of the whole liver.

Patients. The subjects consisted of 4 patients with HCC (4 lesions) who met the aforementioned criteria for treatment, underwent CyberKnife treatment and were evaluated by image analysis using CEUS prior to and following treatment. In all cases, the therapeutic effects were confirmed by dynamic CT following treatment. The patients consisted of 3 males and 1 female, with a mean age of 78.3 years. The underlying liver diseases were hepatitis $\mathrm{C}$ in 3 patients and alcoholic hepatitis in 1 patient. Child-Pugh liver function was class $\mathrm{A}$ in all subjects.
Primary lesions were detected in 2 patients, while distant recurrences were detected in the others. HCC was diagnosed as classical HCC or distant recurrence by CEUS, dynamic CT and gadolinium ethoxybenzyl diethylenetriamine pentaacetic acid-magnetic resonance imaging (MRI). Informed consent was obtained from all patients for inclusion in this study.

CEUS methodology. CEUS was performed prior to treatment, at 2 and 4 weeks post-CyberKnife treatment, and every 4 weeks thereafter for as long as possible. Evaluation by CEUS was completed if the therapeutic effect was confirmed by dynamic CT. The ultrasound equipment used in this examination was an SSA-790A (Toshiba Medical Systems, Tokyo, Japan) with a convex probe (PVT-375BT; 3.75-MHz center frequency). The imaging mode used was wide-band harmonic imaging (pulse subtraction) with transmission/reception frequencies of 1.8 and $3.5 \mathrm{MHz}$, respectively. The mechanical index for acoustic output was set to 0.2 and the dynamic range was set to $60-65 \mathrm{~dB}$. A single focal point was set at the deep site of the lesion, and an intravenous bolus injection of Sonazoid (0.5 ml; Daiichi Sankyo, Tokyo, Japan) was administered via a left cubital venous line followed by flushing with $10 \mathrm{ml}$ of normal saline. Following injection of Sonazoid, the lesion was evaluated with regard to the following: The dynamics of the enhancement of the tumor and the hepatic parenchyma surrounding the tumor in the vascular phase $(0-40 \mathrm{sec})$, and the presence or absence of a hypoechoic area in the hepatic parenchyma surrounding the tumor in the post-vascular phase (after $10 \mathrm{~min}$ ). Subsequently, the dynamics of the enhancement of the tumor and the hepatic parenchyma surrounding the tumor were re-evaluated using the re-injection method (12) with Sonazoid in the post-vascular phase. Digital cine clips of the CEUS images were stored on the hard disk of the scanner and transferred to a high-performance personal computer for subsequent analysis. This study was approved by the Ethical Review Board of Toho University Medical Center, Omori Hospital.

\section{Results}

All HCC lesions showed hyperenhancement by CEUS prior to treatment (Table I). In case 1, the tumor showed hypoenhancement at 40 weeks post-treatment. Strong hyperenhancement was observed in the hepatic parenchyma surrounding the tumor in the vascular phase at 12 weeks post-treatment and a hypoechoic area was observed in the post-vascular phase at 20 weeks post-treatment (Fig. 1). In case 2, the tumor showed hypoenhancement at 8 weeks post-treatment. Strong hyperenhancement was observed in the hepatic parenchyma surrounding the tumor in the vascular phase at 12 weeks post-treatment and a hypoechoic area was observed in the post-vascular phase at 16 weeks post-treatment. In case 3, the tumor showed hypoenhancement at 4 weeks post-treatment (Fig. 2). Strong hyperenhancement was observed in the hepatic parenchyma surrounding the tumor in the vascular phase at 2 weeks post-treatment and a hypoechoic area was observed in the post-vascular phase at 4 weeks post-treatment. In case 4 , the tumor showed hypoenhancement at 12 weeks post-treatment. Strong hyperenhancement was observed in the hepatic parenchyma surrounding the tumor in the vascular 
Table I. Characteristics of patients, and the changes in the hemodynamics of the tumor and the hepatic parenchyma surrounding the tumor, as observed by CEUS.

\begin{tabular}{|c|c|c|c|c|c|c|c|c|}
\hline \multirow[b]{2}{*}{ Case } & \multirow[b]{2}{*}{ Etiology } & \multirow[b]{2}{*}{$\begin{array}{l}\text { Child-Pugh } \\
\text { classification }\end{array}$} & \multirow[b]{2}{*}{ Area } & \multirow[b]{2}{*}{$\begin{array}{l}\text { Diameter, } \\
\mathrm{mm}\end{array}$} & \multirow{2}{*}{$\begin{array}{l}\text { Amount of } \\
\text { radiation, } \\
\text { Gy }\end{array}$} & \multirow[b]{2}{*}{$\begin{array}{l}\text { Week of tumor } \\
\text { hypoenhancement }{ }^{\mathrm{a}}\end{array}$} & \multicolumn{2}{|c|}{$\begin{array}{l}\text { Hepatic parenchyma } \\
\text { surrounding the tumor }\end{array}$} \\
\hline & & & & & & & $\begin{array}{c}\text { Week of } \\
\text { hyperenhancement }\end{array}$ & $\begin{array}{c}\text { Week of } \\
\text { hypoechoic areac }\end{array}$ \\
\hline 1 & $\mathrm{HCV}$ & $5-\mathrm{A}$ & S6 & 24 & 50 & 40 & 12 & 20 \\
\hline 2 & $\mathrm{HCV}$ & 6-A & S8 & 37 & 36 & 8 & 12 & 16 \\
\hline 3 & Alcohol & $5-\mathrm{A}$ & S8 & 12 & 54 & 4 & 2 & 4 \\
\hline 4 & $\mathrm{HCV}$ & $5-\mathrm{A}$ & S3 & 25 & 54 & 12 & 8 & 8 \\
\hline
\end{tabular}

${ }^{\mathrm{a}}$ Onset of the hemodynamic change in the vascular phase of CEUS after CyberKnife ${ }^{\circledR}$ treatment. ${ }^{\mathrm{b}}$ Onset of the hyperenhancement area appearance in the vascular phase of CEUS after CyberKnife treatment. 'Onset of the hypoechoic area appearance in the post-vascular phase of CEUS after CyberKnife treatment. $\mathrm{HCV}$, hepatitis C virus; CEUS, contrast-enhanced ultrasonography.
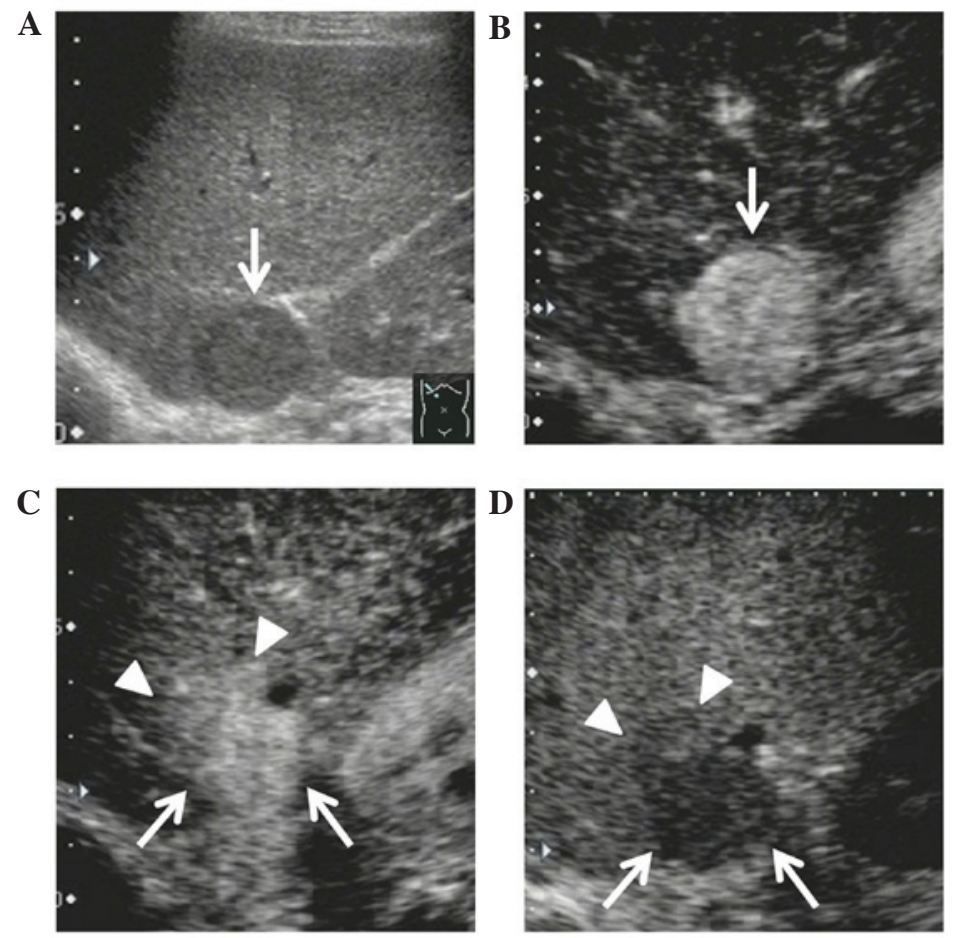

Figure 1. Case 1. (A) Gray-scale US showing a hypoechoic lesion of $24 \mathrm{~mm}$ in diameter in S6 prior to treatment (arrow). (B) CEUS showing hyperenhancement of the tumor in the vascular phase prior to treatment (arrow). (C) CEUS showing strong hyperenhancement of the tumor (arrow) and the hepatic parenchyma surrounding the tumor (arrowhead) in the vascular phase at 12 weeks post-treatment and (D) a hypoechoic area of the tumor (arrow) and the hepatic parenchyma surrounding the tumor (arrowhead) in the post-vascular phase at 20 weeks post-treatment. US, ultrasonography; CEUS, contrast-enhanced ultrasonography.

phase at 8 weeks post-treatment and a hypoechoic area was observed in the post-vascular phase at 8 weeks post-treatment.

The results can be summarized as follows: i) In the patient with earlier changes, hemodynamic changes were evident in the tumor at 4 weeks and in the hepatic parenchyma surrounding the tumor at 2 weeks post-treatment, respectively; ii) the tumor showed hypoenhancement in all patients; and iii) with regard to findings in the hepatic parenchyma surrounding the tumor, strong hyperenhancement appeared in the vascular phase initially, followed by a hypoechoic area in the post-vascular phase.

\section{Discussion}

We previously evaluated intratumoral hemodynamics during progression along the multistep pathway of HCC (13) and early responses to sorafenib for HCC using CEUS with Sonazoid. It was confirmed that CEUS with Sonazoid was less invasive compared with dynamic CT, due to the lack of iodine allergy, exposure to radiation and influence of renal function by administration of the contrast agent. Furthermore, a detailed evaluation, based on the observation of real-time hemodynamics following injection of Sonazoid, was provided. In 

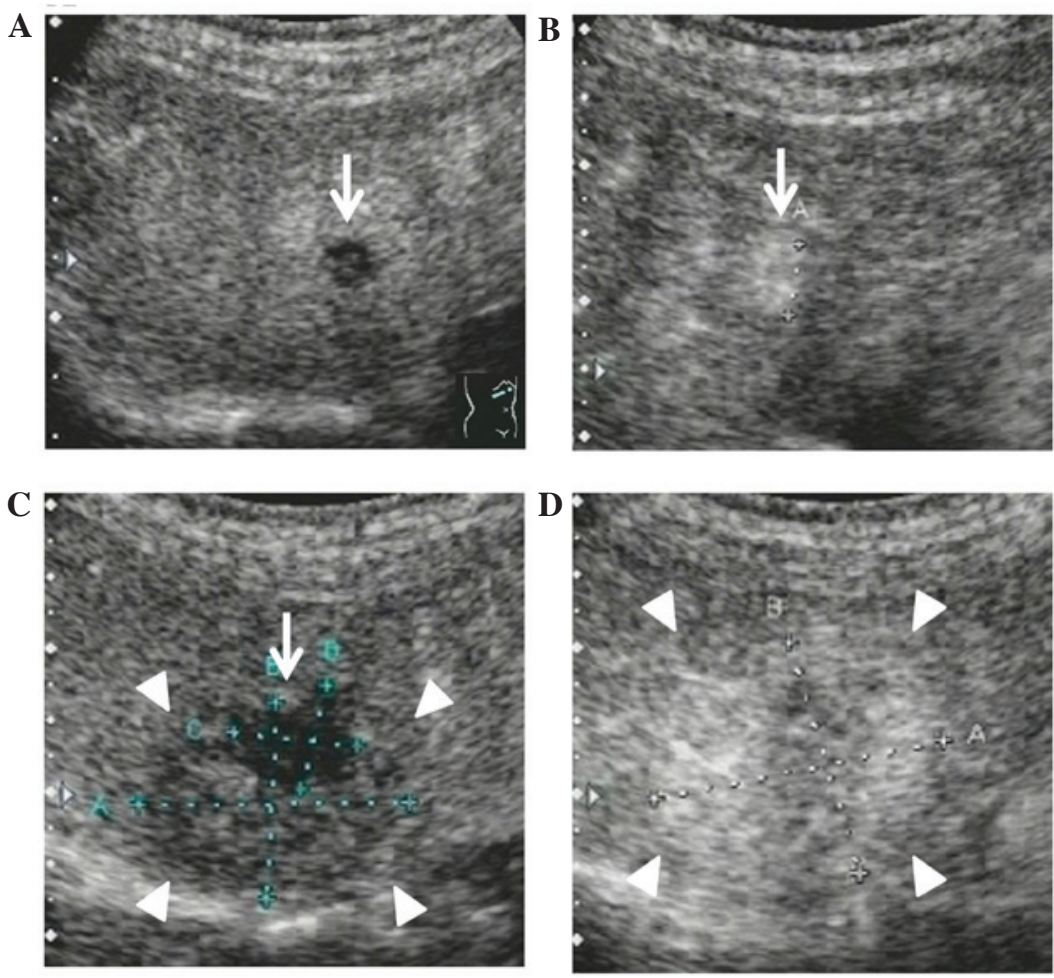

Figure 2. Case 3. (A) CEUS showing a hypoechoic area of $12 \mathrm{~mm}$ in diameter in S8 in the post-vascular phase prior to treatment (arrow). (B) CEUS using the re-injection method showing hyperenhancement of the tumor prior to treatment (arrow). (C) CEUS showing a hypoechoic area of the tumor (arrow) and the hepatic parenchyma surrounding the tumor (arrowhead) in the post-vascular phase at 4 weeks post-treatment. (D) CEUS using the re-injection method showing strong hyperenhancement of the tumor and the hepatic parenchyma surrounding the tumor (arrowhead) in the vascular phase at 4 weeks post-treatment. CEUS, contrast-enhanced ultrasonography.

addition, CEUS was useful for determining the early responses to sorafenib for HCC $(11,14)$.

CyberKnife is expected to be a novel local treatment for HCC, however, it takes a long-term follow-up to determine the effect of treatment in a number of the affected patients when using dynamic CT and MRI (15). Therefore, there is a requirement to evaluate procedures for early determination of the effect of CyberKnife treatment. The hemodynamics of liver metastases and hepatic parenchyma surrounding the tumors in SBRT have been reported on CEUS with SonoView (Bracco, Milan, Italy) (16). However, there has been no report of the use of CEUS with Sonazoid to examine the hemodynamics during and following CyberKnife treatment for HCC. In the present study, CEUS with Sonazoid was performed prior to and following CyberKnife treatment for HCC patients, and the therapeutic applicability of CEUS with Sonazoid was evaluated for the early determination of the effect of CyberKnife treatment.

Although the appearance of the hemodynamic changes in the tumor and the hepatic parenchyma surrounding the tumor varied depending on the patient in this study, in the patient with earlier changes, the hemodynamic changes were evident in the tumor at 4 weeks and in the hepatic parenchyma surrounding the tumor at 2 weeks post-treatment, respectively. These differences in the timing of the appearance in hemodynamic changes following treatment between patients were affected by the differences in the tumor size and site, the total irradiation dose and the liver function.

The tumors showed hypoenhancement in all patients, despite differences in the timing of their appearance. In HCC after radiotherapy, contrast enhancement is considered to persist for a relatively long period. By contrast, it has been reported that perfusion in the tumor correlates with tumor vitality and vascularization (17). Changes in tumor vascularization during or following treatment may be prognostic factors, and reduction of tumor vascularization was considered to indicate a therapeutic effect.

With regard to the findings in the hepatic parenchyma surrounding the tumor, strong hyperenhancement appeared initially in the vascular phase, followed by hypoechoic areas in the post-vascular phase of each patient in the present study. These findings are considered to indicate radiation-induced liver disease, based upon the findings in the study by Reed and Cox (18). This study examined histological changes in radiation-induced liver disease and confirmed the association with the histological changes of veno-occlusive disease, suggesting that the cause was vascular endothelial dysfunction caused by the irradiation (18). The hepatic vein, distinct from the hepatic artery and the portal vein, may be likely to exhibit damage in the vascular endothelium due to the absence of Glisson's capsule, resulting in congestion of the hepatic parenchyma and damage to the hepatic vein. Consequently, an arterio-portal shunt occurred, which was observed as strong hyperenhancement in this study. Furthermore, damaged Kupffer cells induced dysfunction, which likely appeared as hypoechoic areas in the area that showed strong hyperenhancement during the post-vascular phase (18).

Radiation-induced liver disease is generally considered to develop at 4-8 weeks post-treatment (19). On the other hand, 
CT images, which are most frequently used for the evaluation of radiation-induced liver disease, have shown that the median timing for the confirmation of radiation liver disorder is 3 months (15). In the present study and a previous study of liver metastases using CEUS with SonoView (16), changes of hemodynamics in the hepatic parenchyma surrounding the tumor were detected comparatively earlier than those on CT images. The reason was that CEUS was sensitive to the evaluation of hemodynamics and was less invasive. Therefore, frequent testing with CEUS can demonstrate earlier changes after treatment.

It would be premature to conclude that all the hemodynamic changes in the hepatic parenchyma surrounding the tumor after CyberKnife treatment reflect the therapeutic effect against HCC due to of the small scale of the present study. However, the strong hyperenhancement observed in the hepatic parenchyma surrounding the tumor may correspond with the irradiation field. Therefore, if these findings are detected early after treatment, the tumor may be irradiated sufficiently. It has been suggested that CEUS may be applicable to the early determination of the therapeutic effect of CyberKnife treatment in combination with the evaluation of intratumoral vascularization. Further investigations should be conducted in large-scale studies to support this hypothesis.

In conclusion, evaluation of the hemodynamics of tumors and the hepatic parenchyma surrounding the tumor using CEUS with Sonazoid may be therapeutically applicable, as it is less invasive and provides an early evaluation of the effectiveness of CyberKnife treatment.

\section{Acknowledgements}

The authors wish to thank medical technologists Mr. Takehide Kudo and Mr. Kenichi Maruyama of the Department of Clinical Functional Physiology, Toho University Medical Center, Omori Hospital.

\section{References}

1. Ernst F, Schlaefer A and Schweikard A: Smoothing of respiratory motion traces for motion-compensated radiotherapy. Med Phys 37: 282-294, 2010.

2. Martin A and Gaya A: Stereotactic body radiotherapy: A review. Clin Oncol (R Coll Radiol) 22: 157-172, 2010.

3. Adler JR Jr, Chang SD, Murphy MJ, Doty J, Geis P and Hancock SL: The Cyberknife: A frameless robotic system for radiosurgery. Stereotact Funct Neurosurg 69: 124-128, 1997.
4. Louis C, Dewas S, Mirabel X, Lacornerie T, Adenis A, Bonodeau $F$ and Lartigau E: Stereotactic radiotherapy of hepatocellular carcinoma: Preliminary results. Technol Cancer Res Treat 9: 479-487, 2010.

5. Yuan ZY, Meng MB, Liu CL, et al: Stereotactic body radiation therapy using the CyberKnife ${ }^{\circledR}$ system for patients with liver metastases. Onco Targets Ther 7: 915-923, 2014.

6. Dewas S, Bibault JE, Mirabel X, et al: Prognostic factors affecting local control of hepatic tumors treated by stereotactic body radiation therapy. Radiat Oncol 7: 166, 2012.

7. Yuan Z, Tian L, Wang P, Song Y, Dong Y and Zhuang H: Comparative research on the efficacy of CyberKnife ${ }^{\circledR}$ and surgical excision for Stage I hepatocellular carcinoma. Onco Targets Ther 6: 1527-1532, 2013.

8. Bibault JE, Dewas S, Vautravers-Dewas C, Hollebecque A, Jarraya H, Lacornerie T, Lartigau E and Mirabel X: Stereotactic body radiation therapy for hepatocellular carcinoma: Prognostic factors of local control, overall survival, and toxicity. PLoS One 8: e77472, 2013

9. Que JY, Lin LC, Lin KL, Lin CH, Lin YW and Yang CC: The efficacy of stereotactic body radiation therapy on huge hepatocellular carcinoma unsuitable for other local modalities. Radiat Oncol 9: 120, 2014.

10. Watanabe M, Shiozawa K, Takahashi M, Wakui N, Otsuka Y, Kaneko H, Tanikawa K, Shibuya K, Kamiyama N and Sumino Y: Parametric imaging using contrast-enhanced ultrasound with Sonazoid for hepatocellular carcinoma. J Med Ultrason (2001) 37: 81-86, 2010

11. Shiozawa K, Watanabe M, Kikuchi Y, Kudo T, Maruyama K and Sumino Y: Evaluation of sorafenib for hepatocellular carcinoma by contrast-enhanced ultrasonography: A pilot study. World J Gastroenterol 18: 5753-5758, 2012.

12. Kudo M: Hepatocellular carcinoma 2009 and beyond: From the surveillance to molecular targeted therapy. Oncology 75: 1-12, 2008.

13. Matsui O, Kadoya M, Kameyama T, Yoshikawa J, Takashima T, Nakanuma Y, Unoura M, Kobayashi K, Izumi R and Ida M: Benign and malignant nodules in cirrhotic livers: Distinction based on blood supply. Radiology 178: 493-497, 1991.

14. Shiozawa K, Watanabe M and Sumino Y: Evaluation of the hemodynamic status of focal hepatic lesions $20 \mathrm{~mm}$ or less in diameter by contrast-enhanced ultrasonography using Sonazoid. Intervirology 52: 213-222, 2009.

15. Sanuki-Fujimoto N, Takeda A, Ohashi T, Kunieda E, Iwabuchi S, Takatsuka K, Koike N and Shigematsu N: CT evaluations of focal liver reactions following stereotactic body radiotherapy for small hepatocellular carcinoma with cirrhosis: Relationship between imaging appearance and baseline liver function. Br J Radiol 83: 1063-1071, 2010

16. Krix M, Plathow C, Essig M, Herfarth K, Debus J, Kauczor HU and Delorme S: Monitoring of liver metastases after stereotactic radiotherapy using low-MI contrast-enhanced ultrasound - initial results. Eur Radiol 15: 677-684, 2005.

17. Weidner N, Semple JP, Welch WR and Folkman J: Tumor angiogenesis and metastasis--correlation in invasive breast carcinoma N Engl J Med 324: 1-8, 1991.

18. Reed GB Jr and Cox AJ Jr: The human liver after radiation injury. A form of veno-occlusive disease. Am J Pathol 48: 597-611, 1966.

19. Lawrence TS, Robertson JM, Anscher MS, Jirtle RL, Ensminger WD and Fajardo LF: Hepatic toxicity resulting from cancer treatment. Int J Radiat Oncol Biol Phys 31: 1237-1248, 1995. 